\title{
Gyroscopic Forces and Collision Avoidance with Convex Obstacles
}

\author{
Dong Eui Chang ${ }^{1}$ and Jerrold E. Marsden ${ }^{2}$ \\ 1 Mechanical \& Environmental Engineering, University of California, Santa \\ Barbara, CA 93106-5070; dchang@engineering.ucsb.edu \\ 2 Control \& Dynamical Systems, California Institute of Technology, Pasadena, CA \\ 91125; marsden@cds. caltech.edu
}

Summary. This paper introduces gyroscopic forces as an tool that can be used in addition to the use of potential forces in the study of collision and convex obstacle avoidance. It makes use of the concepts of a detection shell and a safety shell and shows, in an appropriate context, that collisions are avoided, while at the same time guaranteeing that control objectives determined by a potential function are met. In related publications, we refine and extend the method to include flocking and swarming behavior.

\section{Introduction}

Goals of the Paper. The purpose of this paper is to make use of the techniques of controlled Lagrangians given in [3] and references therein - in particular gyroscopic control forces - in the problem of collision and obstacle avoidance. We are also inspired by the work of Wang and Krishnaprasad [9]. An interesting feature of gyroscopic forces is that they do not interfere with any prior use of potential forces, as in the fundamental work on the navigation function method of Rimon and Koditschek [8], that may have been set up for purposes of setting control objectives. In particular, the method avoids the often encountered difficulty of purely potential theoretic methods in which unwanted local minima appear. The techniques we develop appear to be efficient and the algorithms provably respect given safety margins.

This paper is a preliminary report on the methodology of gyroscopic forces. We will be developing it further in the future in the context of networks of agents, including underwater vehicles and other systems. Of course these agents have nontrivial internal dynamics that need to be taken into account, but our view (consistent with methods developed by Steve Morse - see, for instance, [6]) is that only information concerning a "safety shell" need be transmitted to the vehicle network, and this perhaps only to nearest neighbors, rather than all the detailed state information about each agent. Of course such 
a hierarchical and networked approach is critical for a strategy of this sort to be scalable.

Collision avoidance is of course a key ingredient in coordinated control of vehicles and in particular in the flight control community. We refer to, for example, [5]. Earlier work, such as [7] introduces "vortical" forces that are reminiscent of, but not the same as, gyroscopic forces studied in the present paper. A future goal is to apply the present method to coordinated control of groups of underwater vehicles; see, for instance, [1] and references therein.

The present paper was inspired by a Caltech lecture of Elon Rimon and we thank him for very useful conversations about the subject and his interest. While there remain a number of important results which remain to be proved in the present context, we hope that the work described here will be helpful towards incorporating gyroscopic forces more systematically into methods based on potential functions. The techniques are further developed in [4] and applied to the problem of flocking and swarming behavior.

Gyroscopic Forces. Gyroscopic forces denote forces which do not do any work. Mathematically, a force $F_{g}$ defined to be a gyroscopic force if $F_{g} \cdot \dot{\mathbf{q}}=0$ where $\dot{\mathbf{q}}$ is a velocity vector. A general class of gyroscopic force $F_{g}$ have the form

$$
F_{g}=S(\mathbf{q}, \dot{\mathbf{q}}) \dot{\mathbf{q}}
$$

where $S$ is a skew symmetric matrix. There are two useful viewpoints on gyroscopic forces in the dynamics of mechanical systems. One is that gyroscopic forces create coupling between different degrees of freedom, just like mechanical couplings. The other is that gyroscopic forces rotate the velocity vector just like a magnetic field acting on a charged particle. The first interpretation regards the matrix $S$ in (1) as an interconnection matrix and the second interpretation considers $S$ as an infinitesimal rotation. In this paper, we will take the second viewpoint and use gyroscopic forces to prevent vehicles from colliding with obstacles or other vehicles. In the future, we will also elaborate on the first viewpoint relating the matrix $S$ to the graph of inter-vehicle communication links. The first viewpoint was taken in [3] when gyroscopic forces were introduced into the method of controlled Lagrangian; indeed, gyroscopic forces are very useful in stabilization of mechanical systems.

\section{Obstacle Avoidance}

The problem of obstacle avoidance is important in robotics and multivehicle systems. The objective is to design a controller for a robot so that it approaches its target point without colliding with any obstacles during the journey. We will employ potential forces, dissipative forces, and gyroscopic forces. The first two forces take care of convergence to the target point and the gyroscopic force handles the obstacle avoidance. We will compare our 
method with the navigation function method which was developed in [8]. For the sake of easy exposition, we address a particular situation where there is only one obstacle in a plane. Since our algorithm uses only local information around the vehicle, the same control law works for multiple obstacles.

Obstacle Avoidance by Gyroscopic Forces. Suppose that there is a fully actuated vehicle and an obstacle in the $x y$-plane. For the purpose of exposition, we assume that the vehicle is a point of unit mass and the obstacle is a unit disk located at the origin. We want to design a feedback control law to (asymptotically) drive the vehicle to a target point $\mathbf{q}_{T}=\left(x_{T}, y_{T}\right)$ without colliding with the obstacle. A detection shell, a ball of radius $r_{\text {det }}$ is given to the vehicle such that the vehicle will respond to the obstacle only when the obstacle comes into the detection shell. Safety shells can be readily added to this discussion, as in $\S 3$ below; the safety shell itself is designed to not collide with the obstacle.

The dynamics of the vehicle are given simply by $\ddot{\mathbf{q}}=u$, where $\mathbf{q}=(x, y)$ and $u=\left(u_{x}, u_{y}\right)$. The control $u$ consists of four parts as follows:

$$
u=F_{p}+F_{d}+F_{g}+v
$$

where $F_{p}$ is a potential force which assigns to the vehicle a potential function with the minimum at the target $\mathbf{q}_{T} ; F_{d}$ is a dissipative force; $F_{g}$ is a gyroscopic force; and $v$ is an another control force. We set $v$ to zero unless this additional control is needed (as remarked later, it may be useful in near-zero velocity collisions). The three forces, $F_{p}, F_{d}$, and $F_{g}$ are of the following form:

$$
F_{p}=-\nabla V(\mathbf{q}), \quad F_{d}=-D(\mathbf{q}, \dot{\mathbf{q}}) \dot{\mathbf{q}}, \quad F_{g}=S(\mathbf{q}, \dot{\mathbf{q}}) \dot{\mathbf{q}}
$$

where $V$ is a (potential) function on $\mathbb{R}^{2}$, the matrix $D$ is symmetric and positive-definite, and the matrix $S$ is skew-symmetric.

We choose the potential function $V$ and the dissipative force $F_{d}$ as follows:

$$
V(\mathbf{q})=\frac{1}{2}\left\|\mathbf{q}-\mathbf{q}_{T}\right\|^{2}, \quad F_{d}=-2 \dot{\mathbf{q}} .
$$

Before we choose a gyroscopic force, let us introduce some definitions. Let $\mathbf{d}(\mathbf{q})=\left(d_{x}(\mathbf{q}), d_{y}(\mathbf{q})\right)$ be the vector from the vehicle position, $\mathbf{q}$, to the nearest point in the obstacle. Since the obstacle is convex, the vector $\mathbf{d}(\mathbf{q})$ is well defined. Let $d(\mathbf{q})=\|\mathbf{d}(\mathbf{q})\|$ be the distance between the vehicle and the obstacle. We now choose the following gyroscopic force $F_{g}$

$$
F_{g}=\left[\begin{array}{cc}
0 & -\omega(\mathbf{q}, \dot{\mathbf{q}}) \\
\omega(\mathbf{q}, \dot{\mathbf{q}}) & 0
\end{array}\right] \dot{\mathbf{q}}
$$

Here, the function $\omega$ is defined by 


$$
\omega(\mathbf{q}, \dot{\mathbf{q}})=\left\{\begin{array}{cl}
\frac{\pi V_{\max }}{d(\mathbf{q})} & \text { if }\left[d(\mathbf{q}) \leq r_{\mathrm{det}}\right] \wedge[\mathbf{d}(\mathbf{q}) \cdot \dot{\mathbf{q}}>0] \wedge[\operatorname{det}[\mathbf{d}(\mathbf{q}), \dot{\mathbf{q}}] \geq 0] \\
-\frac{\pi V_{\max }}{d(\mathbf{q})} & \text { if }\left[d(\mathbf{q}) \leq r_{\mathrm{det}}\right] \wedge[\mathbf{d}(\mathbf{q}) \cdot \dot{\mathbf{q}}>0] \wedge[\operatorname{det}[\mathbf{d}(\mathbf{q}), \dot{\mathbf{q}}]<0] \\
0 & \text { otherwise }
\end{array}\right.
$$

where $V_{\max }>0$ is a constant and $\wedge$ denotes the logical "and". The meaning of the function $\omega$ is as follows. The vehicle gets turned by the gyroscopic force only when it detects an obstacle in the detection shell $\left(d(\mathbf{q}) \leq r_{\text {det }}\right)$ and it is heading toward the obstacle $(\mathbf{d}(\mathbf{q}) \cdot \dot{\mathbf{q}}>0)$. The role of the gyroscopic force is to rotate the velocity vector (as indicated in (3)). The direction of the rotation (that is, the sign of $\omega(\mathbf{q}, \dot{\mathbf{q}})$ ) depends on the orientation of the two vectors, $\mathbf{d}(\mathbf{q})$ and $\dot{\mathbf{q}}$, i.e, the sign of $\operatorname{det}[\mathbf{d}(\mathbf{q}), \dot{\mathbf{q}}]$.

The energy $E$ of the vehicle is given by its kinetic plus potential energies:

$$
E(\mathbf{q}, \dot{\mathbf{q}})=\frac{1}{2}\|\dot{\mathbf{q}}\|^{2}+V(\mathbf{q}) .
$$

One checks that the energy is non-increasing in time as follows:

$$
\frac{d}{d t} E(\mathbf{q}, \dot{\mathbf{q}})=\dot{\mathbf{q}} \cdot F_{d}=-2\|\dot{\mathbf{q}}\|^{2} \leq 0 .
$$

We now prove, by contradiction, that the vehicle does not collide with the obstacle at nonzero velocity when the initial energy satisfies

$$
E(\mathbf{q}(0), \dot{\mathbf{q}}(0)) \leq \frac{1}{2} V_{\max }^{2},
$$

where $V_{\max }$ is the positive constant in (4). Suppose that the vehicle collided with the obstacle at time $t=t_{c}<\infty$ with velocity $\dot{\mathbf{q}}\left(t_{c}\right) \neq 0$. Take a small $\Delta t>0$ and consider the dynamics in the time interval, $I=\left[t_{c}-\Delta t, t_{c}^{-}\right]$. Without loss of generality, we may assume $\operatorname{det}[\mathbf{d}(\mathbf{q}), \dot{\mathbf{q}}] \geq 0$ in $I$. Then, the dynamics are given by

$$
\ddot{\mathbf{q}}=\left[\begin{array}{cc}
-2 & -\omega(\mathbf{q}, \dot{\mathbf{q}}) \\
\omega(\mathbf{q}, \dot{\mathbf{q}}) & -2
\end{array}\right] \dot{\mathbf{q}}-\left(\mathbf{q}-\mathbf{q}_{T}\right)
$$

with $\omega(\mathbf{q}, \dot{\mathbf{q}})=\pi V_{\max } / d(\mathbf{q})$. One can integrate this ODE for $\dot{\mathbf{q}}$ during $I$ as follows:

$$
\begin{gathered}
\dot{\mathbf{q}}\left(t_{c}^{-}\right)=e^{-2 \Delta t}\left[\begin{array}{cc}
\cos \theta\left(t_{c}^{-}\right)-\sin \theta\left(t_{c}^{-}\right) \\
\sin \theta\left(t_{c}^{-}\right) & \cos \theta\left(t_{c}^{-}\right)
\end{array}\right] \dot{\mathbf{q}}\left(t_{c}-\Delta t\right) \\
\left.-\int_{t_{c}-\Delta t}^{t_{c}^{-}} e^{-2\left(t_{c}^{-}-\tau\right)}\left[\begin{array}{ll}
\cos \left(\theta\left(t_{c}^{-}\right)-\theta(\tau)\right)-\sin \left(\theta\left(t_{c}^{-}\right)-\theta(\tau)\right) \\
\sin \left(\theta\left(t_{c}^{-}\right)-\theta(\tau)\right) & \cos \left(\theta\left(t_{c}^{-}\right)-\theta(\tau)\right)
\end{array}\right]\left(\mathbf{q}(\tau)-\mathbf{q}_{T}\right)\right) d \tau
\end{gathered}
$$

where 


$$
\theta(t)=\int_{t_{c}-\Delta t}^{t} \omega(\mathbf{q}(s), \dot{\mathbf{q}}(s)) d s=\int_{t_{c}-\Delta t}^{t} \frac{\pi V_{\max }}{d(\mathbf{q}(t))} d s .
$$

Since the velocity $\dot{\mathbf{q}}(t)$ is continuous on the time interval $I$, there is a $\beta>0$ such that $\|\dot{\mathbf{q}}(t)\|>\beta$ in $I$. We can therefore rewrite (8) as

$$
\dot{\mathbf{q}}\left(t_{c}^{-}\right)=e^{-2 \Delta t}\left[\begin{array}{cc}
\cos \theta\left(t_{c}^{-}\right) & -\sin \theta\left(t_{c}^{-}\right) \\
\sin \theta\left(t_{c}^{-}\right) & \cos \theta\left(t_{c}^{-}\right)
\end{array}\right] \dot{\mathbf{q}}\left(t_{c}-\Delta t\right)+O(\Delta t) .
$$

because $\|\mathbf{q}(t)\|$ is bounded during $I$ and $\Delta t$ is very small. By (5), (6), (7) and $V(\mathbf{q}) \geq 0$, we have $\|\dot{\mathbf{q}}(t)\| \leq V_{\max }$ for $t \in I$. So,

$$
\Delta t \geq \frac{d\left(\mathbf{q}\left(t_{c}-\Delta t\right)\right)}{V_{\max }}
$$

Since the trajectory is approaching the obstacle during $I$, one may assume that

$$
d(\mathbf{q}(t)) \leq d\left(\mathbf{q}\left(t_{c}-\Delta t\right)\right)
$$

for $t \in I$. It follows from (9), (11) and (12) that

$$
\theta\left(t_{c}^{-}\right) \geq \pi
$$

Notice that the inequality (13) is independent of $\Delta t$. We can conclude from (10) and (13) that the velocity vector $\dot{\mathbf{q}}(t)$ rotates more than, say, $3 \pi / 4$ radians during the interval $\left[t_{c}-\Delta t, t_{c}^{-}\right]$for a small $\Delta t>0$. However, since we assumed that the vehicle collided with the obstacle at $t=t_{c}$ with nonzero velocity, the velocity cannot rotate much during the interval $\left[t_{c}-\Delta t, t_{c}^{-}\right]$for a small $\Delta t>0$ by the continuity of $\dot{\mathbf{q}}(t)$. We have reached a contradiction and therefore, there is no (finite-time) collision of the vehicle with the obstacle at nonzero velocity.

There are two ways that the vehicle may collide with the obstacle: in a finite time or in infinite time. As shown above, a finite-time collision occurs only if

$$
\dot{\mathbf{q}}\left(t_{c}\right)=0
$$

where $t_{c}$ is the moment of collision. Let us consider the case where there is a time sequence $t_{i} \nearrow \infty$ such that $\mathbf{q}\left(t_{i}\right)$ converges to the obstacle. By $(5)$ $\operatorname{and}(6)$,

$$
\int_{0}^{\infty}\|\dot{\mathbf{q}}(\tau)\|^{2} d \tau \leq \frac{1}{2} E(0)<\infty
$$

Hence, there exists a time sequence $s_{i} \nearrow \infty$ such that

$$
\lim _{i \rightarrow \infty} \dot{\mathbf{q}}\left(s_{i}\right)=0 \text {. }
$$


This means the vehicle slows down, at least sporadically. The common phenomenon in both of these collision possibilities is that the vehicle slows down as shown in (14) and (15). Let us call both types of collision a zero-velocity collision for the sake of simple terminology. One might introduce an additional adaptive control scheme through $v$ in (2) to approach the target as well as avoid the zero-velocity collision. That is, if one assumes that there is an additional control that maintains a minimum velocity, then these zero velocity collision situations can be avoided.

We now discuss the asymptotic convergence of the vehicle to the target in the case that the vehicle does not end up with a zero-velocity collision. Suppose that the trajectory $(\mathbf{q}(t), \dot{\mathbf{q}}(t))$ satisfies (7) and does not end with a zerovelocity collision. Since $\mathbf{q}(t)$ is a certain distance away from the obstacle, there exists an open set $W \subset \mathbb{R}^{2}$ containing the obstacle such that the trajectory lies in the compact set

$$
K:=E^{-1}([0, E(t=0)]) \backslash\left(W \times \mathbb{R}^{2}\right) .
$$

Then, the trajectory exists for all $t \geq 0$. One can adapt the usual version of LaSalle's invariance principle to show the asymptotic convergence of the trajectory to the target state, $\left(\mathbf{q}_{T}, 0\right)$, where the energy in (5) is used as a Lyapunov function. Here, we give an alternative proof of convergence. Consider the following function:

$$
\begin{aligned}
U(\mathbf{q}, \dot{\mathbf{q}}) & =E(\mathbf{q}, \dot{\mathbf{q}})+\epsilon \mathbf{d} V \cdot \dot{\mathbf{q}} \\
& =\frac{1}{2}\left(\dot{x}^{2}+\dot{y}^{2}+\left(x-x_{T}\right)^{2}+\left(y-y_{T}\right)^{2}\right)+\epsilon\left(\left(x-x_{T}\right) \dot{x}+\left(y-y_{T}\right) \dot{y}\right)
\end{aligned}
$$

with $0<\epsilon<1$. See the Appendix for the motivation for the above choice of Lyapunov function. One can check that (a) $U\left(\mathbf{q}_{T}, 0\right)=0$ and $U(\mathbf{q}, \dot{\mathbf{q}})>0$ on $K \backslash\left\{\left(\mathbf{q}_{T}, 0\right)\right\}$, and (b) $\left(\mathbf{q}_{T}, 0\right)$ is the only critical point of $U$ on $K$. Along the trajectory,

$$
\begin{aligned}
\frac{d U}{d t}= & -(2-\epsilon)\|\dot{\mathbf{q}}\|^{2}-\epsilon\left\|\mathbf{q}-\mathbf{q}_{T}\right\|^{2}-2 \epsilon\left(\mathbf{q}-\mathbf{q}_{T}\right) \cdot \dot{\mathbf{q}} \\
& +\epsilon\left(\mathbf{q}-\mathbf{q}_{T}\right)\left[\begin{array}{cc}
0 & -\omega(\mathbf{q}, \dot{\mathbf{q}}) \\
\omega(\mathbf{q}, \dot{\mathbf{q}}) & 0
\end{array}\right] \dot{\mathbf{q}} .
\end{aligned}
$$

Since $\omega(\mathbf{q}, \dot{\mathbf{q}})$ is bounded on $K$, one can find $\epsilon>0$ and $c>0$ such that

$$
\frac{d U}{d t} \leq-c U \leq 0
$$

on $K$. It follows that $U(t) \leq U(0) e^{-c t}$. This proves that the trajectory asymptotically converges to the target.

In summary, we have shown that the vehicle semi-globally converges to the target state without collision with the obstacle except possibly for a zerovelocity collision. Here, the semi-global property comes from the dependence of 
$V_{\max }$ on the initial condition given in (7). We may avoid zero-velocity collision by adding an adaptive scheme. We expect that the set of initial states ending up with zero-velocity collision is small.

\section{Remarks.}

1. The choice of $V_{\max }$ satisfying (7) may be conservative. Recall that the gyroscopic force gets turned on only when an obstacle comes into the detection shell. So, we can choose a new value of $V_{\max }$ satisfying $E\left(t=t_{d}\right) \leq \frac{1}{2} V_{\max }^{2}$ at the moment, $t=t_{d}$, when the vehicle detects the obstacle. The proof given above is still valid with this update rule of $V_{\max }$. In this sense, the above collision avoidance algorithm works globally. Moreover, the same control law works in the existence of multiple obstacles since our control law is feedback and the vehicle only uses the local information in its detection shell.

2. One can easily modify the above control algorithm for convex obstacles. When the obstacle is not convex, one needs to add an adaptive scheme. One conservative way is to make a convex buffer shell which contains the nonconvex obstacle and regard this convex shell as an obstacle. However, this entails that the vehicle knows the global shape of the obstacle. In reality, the vehicle may only have a local information of the obstacle. In such a case, one needs to apply a scheme to find a convex arc (or, surface) which divides the detection shell so that the vehicle lies on one side and the obstacle on the other side. Then, one regards this convex arc as an obstacle.

For obstacles and bodies with sharp corners and flat surfaces or edges, the algorithm also needs to be modified; this can be done and will appear in forthcoming works of the authors. We illustrate the results of such an algorithm in Figure 1 below.

3 . We give an alternative choice of a gyroscopic force, which produces a faster convergence of a vehicle to its target point than that in (3). Assume that the vehicle has detected an obstacle in its detection shell. In such a case, let us define the function, $\sigma_{\mathbf{q}_{T}}=\sigma_{\mathbf{q}_{T}}(\mathbf{q})$ as follows:

$$
\sigma_{\mathbf{q}_{T}}(\mathbf{q})=\left\{\begin{array}{l}
0 \text { if the obstacle does not lie between the two points, } \mathbf{q} \text { and } \mathbf{q}_{T} \\
1 \text { otherwise }
\end{array}\right.
$$

where $\mathbf{q}$ is the position of the vehicle and $\mathbf{q}_{T}$ is the target point of the vehicle. Roughly speaking, the function $\sigma_{\mathbf{q}_{T}}$ checks if the vehicle can directly see the target. The new gyroscopic force, $\tilde{F}_{g}$, is defined by the product of the function $\sigma_{\mathbf{q}_{T}}$ and the old gyroscopic function $F_{g}$ in (3) as follows:

$$
\tilde{F}_{g}=\sigma_{\mathbf{q}_{T}} F_{g} .
$$

The vehicle switches off the gyroscopic force if the vehicle can directly see the target even when there is an obstacle nearby. Simulation studies show that this new gyroscopic force gives faster convergence to the target. We expect that the gyroscopic force $\tilde{F}_{g}$ reduces the possibility of a zero-velocity collision.

4. If $\mathbf{d}(\mathbf{q}) \cdot \dot{\mathbf{q}} \approx 0$ and there is a measurement error, then the sign of $\omega(\mathbf{q}, \dot{\mathbf{q}})$ becomes fragile. In such a case, one can choose a constant sign of $\omega(\mathbf{q}, \dot{\mathbf{q}})$ for 
a period. The reason is as follows. The condition, $\mathbf{d}(\mathbf{q}) \cdot \dot{\mathbf{q}} \approx 0$, means that the velocity is almost perpendicular to the obstacle. Hence, the direction of rotation the velocity does not matter much as long as one keeps rotating it until the measurement of the direction of velocity becomes relevant to the vector $\mathbf{d}(\mathbf{q})$ in a certain way. Another possible option is to choose the sign of $\omega$ which agrees with the current direction of the potential plus dissipative force, $-\nabla V+F_{d}$. Issues such as this are important for robustness.

5. Above, we chose a particular form of potential function, dissipative force, and gyroscopic force. However, one can modify the above proof for more general from of $V, F_{d}$ and $F_{g}$. We also assumed that the vehicle is a point mass. In reality, it has a volume. In this case, the vehicle is equipped with two shells around it where the inner shell is the safety shell which contains the vehicle and the outer shell is the detection shell. In this case, one must prevent the obstacle from coming into the safety shell. For example, $d(\mathbf{q})$ in (4) should be modified to the distance from the safety shell to the obstacle.

6. One can extend this control algorithm to three dimensions. In such a case, the skew-symmetric matrix $S$ in the gyroscopic force $F_{g}=S(\mathbf{q}, \dot{\mathbf{q}}) \dot{\mathbf{q}}$ should be an infinitesimal rotation with the axis in parallel to the vector $\mathbf{d}(\mathbf{q}, \dot{\mathbf{q}}) \times \dot{\mathbf{q}}$ when $\mathbf{d}(\mathbf{q}, \dot{\mathbf{q}}) \times \dot{\mathbf{q}} \neq 0$. When $\mathbf{d}(\mathbf{q}, \dot{\mathbf{q}}) \times \dot{\mathbf{q}}=0$, one just chooses a preferred rotational direction, as in the planar case.

Comparison with the Navigation Function Method. We compare our method with the navigation function method developed in [8]. In the navigation function method, when the vehicle is fully actuated and there are some obstacles, then one first designs a potential function which has maxima on the boundary of obstacles and the minimum at the target point where no other local minima are allowed, but saddle points are allowed in dynamics because the stable manifolds of saddle points are measure zero. The control force is the sum of the potential force from the potential function and a dissipative force. Then, the vehicle converges to its target point avoiding collision with obstacles. A caveat in the navigation function method is that the construction of such a potential function depends on the global topology of the configuration space excluding obstacles. In other words, the vehicle must know all the information of obstacles in advance; of course one could also consider developing a more local formulation of the navigation function methodology.

Our method differs fundamentally from the navigation function method in which the potential force is used for both convergence and collision-avoidance. Our method employs a potential force only for convergence and uses a gyroscopic force for collision avoidance. We design our potential function without considering the configuration of obstacles, so it is easy to choose a potential function. We only use local information inside the detection shell of the vehicle to execute the gyroscopic force. Hence, we need not know all the information of obstacles in advance. In either method, one must be careful about the (perhaps remote) possibility of zero-velocity collisions. In general, we regard these two methods as complementary to each other. 
Simulation: One Vehicle + Two Obstacles. Consider the case of one vehicle and two obstacles. One obstacle is a disk of radius 1 located at $(0,0)$ and the other is a disk of radius 2 centered at $(5,0)$ (left side of Figure 1).
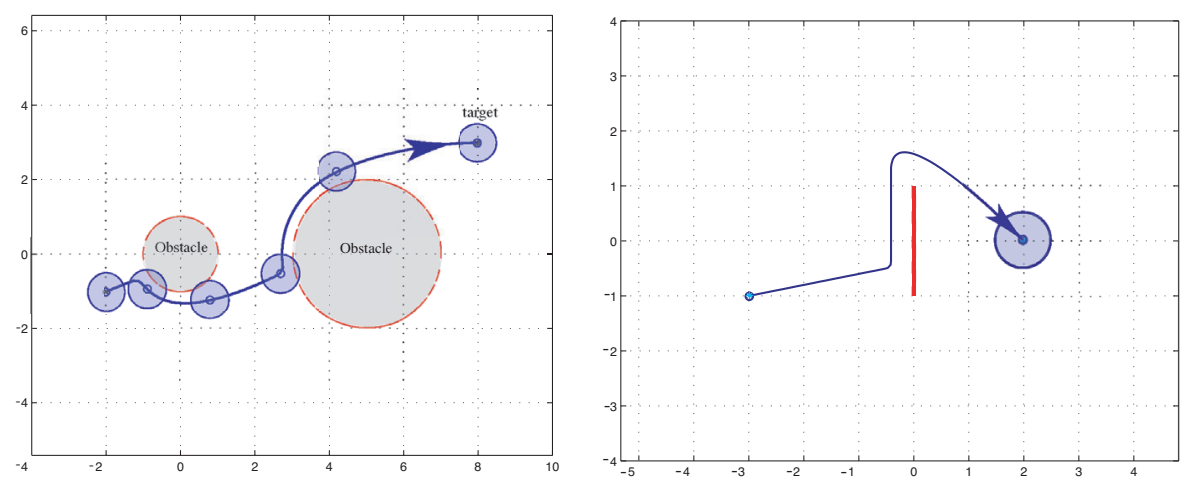

Fig. 1. (Left-Vehicle with two obstacles.) The vehicle starts from $(-2,-1)$ with zero initial velocity, converging to the target point, $(8,3)$, avoiding any collisions with obstacles. The shaded disk about the vehicle is the detection shell. (Right-avoiding a flat obstacle.) This shows a simulation result for a modified algorithm suitable for objects with flat surfaces; the vehicle starts at $(-3,-1)$ and the target is at $(2,0)$.

The vehicle is regarded as a point of unit mass. It starts from $(-2,-1)$ with initial zero velocity and converges to the target point $\mathbf{q}_{T}=(8,3)$. We used the following potential function and dissipative force:

$$
V(\mathbf{q})=\frac{1}{2}\left\|\mathbf{q}-\mathbf{q}_{T}\right\|^{2}, \quad F_{d}=-2 \dot{\mathbf{q}} .
$$

We used $\sqrt{10}$ for $V_{\max }$ in the gyroscopic force in (3) and (4).

The right side of this figure shows that the general methodology, suitably modified also works for objects with flat surfaces, and sharp corners and edges. As mentioned previously, this will be explained in detail in future publications.

\section{Collision Avoidance: Multi-Vehicles}

We develop a collision-avoidance scheme using gyroscopic forces in the case that there are multiple vehicles in a plane. For the purpose of illustration, we only consider two vehicles and we make a remark on how to extend this to multi-vehicle case.

Collision Avoidance between Two Vehicles. Let us consider the situation where there are two vehicles in a plane and there are no other vehicles or 
obstacles. Each vehicle wants to approach its own target point without colliding with the other vehicle. We assume that each vehicle has a finite volume and two shells around its center. The inner shell is called a safety shell, which completely contains the vehicle. Its radius is denoted by $r_{\text {saf }}$. The outer shell is a detection shell of thickness $r_{\text {det }}$. Each vehicle detects the other vehicle only when the other vehicle comes into the detection shell. Here, a collision means the collision between safety shells.

Let $\mathbf{q}_{i}=\left(x_{i}, y_{i}\right)$ be the position of the $i$-th vehicle, and $\mathbf{q}_{T, i}=\left(x_{T, i}, y_{T, i}\right)$ be the target point of the $i$-th vehicle, with $i=1,2$. The dynamics of the $i$-th vehicle are given by $\ddot{\mathbf{q}}_{i}=u_{i}$ with $u_{i}=\left(u_{x_{i}}, u_{y_{i}}\right)$. The control law consists of a potential, a dissipative, and a gyroscopic force: $u=-\nabla V+F_{d}+F_{g}$. For simplicity, we will only design a controller for vehicle 1 in the following. One can get a controller for vehicle 2 in the similar manner.

Choose the following potential function and dissipative force for vehicle 1 :

$$
V_{1}\left(\mathbf{q}_{1}\right)=\frac{1}{2}\left\|\mathbf{q}_{1}-\mathbf{q}_{T, 1}\right\|^{2}, \quad F_{d, 1}\left(\mathbf{q}_{1}, \dot{\mathbf{q}}_{1}\right)=-2 \dot{\mathbf{q}}_{1} .
$$

Before we choose a gyroscopic force, let us define a couple of functions. Let $d\left(\mathbf{q}_{1}, \mathbf{q}_{2}\right)$ be the distance between the safety shells of the two vehicles, which is given by

$$
d\left(\mathbf{q}_{1}, \mathbf{q}_{2}\right)=\left\|\mathbf{q}_{1}-\mathbf{q}_{2}\right\|-r_{\mathrm{saf}, 1}-r_{\mathrm{saf}, 2} .
$$

Define $\varphi: \mathbb{R}^{2} \times \mathbb{R}^{2} \rightarrow[-\pi / 2, \pi / 2]$ by

$$
\varphi(\mathbf{v}, \mathbf{w})= \begin{cases}\text { the signed angle from } \mathbf{v} \text { to } \mathbf{w} & \text { if }[\mathbf{v} \cdot \mathbf{w} \geq 0] \wedge[\|\mathbf{v}\| \cdot\|\mathbf{w}\| \neq 0], \\ 0 & \text { otherwise }\end{cases}
$$

For example, $\varphi((1,0),(1,1))=\pi / 4$ and $\varphi((1,1),(1,0))=-\pi / 4$. Define $\chi$ : $\mathbb{R}^{2} \times \mathbb{R}^{2} \rightarrow \mathbb{R}$ by

$$
\chi\left(\mathbf{q}_{1}, \mathbf{q}_{2}\right)= \begin{cases}1 & \text { if } d\left(\mathbf{q}_{1}, \mathbf{q}_{2}\right) \leq r_{\mathrm{det}, 1} \\ 0 & \text { otherwise }\end{cases}
$$

which checks if vehicle 2 is in the detection shell of vehicle 1 . For the position vectors $\mathbf{q}_{1}$ and $\mathbf{q}_{2}$ of both vehicles, define $\mathbf{q}_{21}=\mathbf{q}_{2}-\mathbf{q}_{1}, \quad \mathbf{q}_{12}=-\mathbf{q}_{21}$. The gyroscopic force $F_{g, 1}$ of vehicle 1 is given by

$$
F_{g, 1}=\chi\left(\mathbf{q}_{1}, \mathbf{q}_{2}\right)\left[\begin{array}{cc}
0 & -\omega\left(\mathbf{q}_{1}, \dot{\mathbf{q}}_{1}, \mathbf{q}_{2}, \dot{\mathbf{q}}_{2}\right) \\
\omega\left(\mathbf{q}_{1}, \dot{\mathbf{q}}_{1}, \mathbf{q}_{2}, \dot{\mathbf{q}}_{2}\right) & 0
\end{array} \dot{\mathbf{q}}_{1}\right.
$$

where the function $\omega$ is given by

$$
\omega\left(\mathbf{q}_{1}, \dot{\mathbf{q}}_{1}, \mathbf{q}_{2}, \dot{\mathbf{q}}_{2}\right)=f\left(\mathbf{q}_{1}, \dot{\mathbf{q}}_{1}, \mathbf{q}_{2}, \dot{\mathbf{q}}_{2}\right) \frac{\pi V_{\max }}{d\left(\mathbf{q}_{1}, \mathbf{q}_{2}\right)}
$$

where $V_{\max }$ is a fixed positive number and the function $f$ is defined by considering four possible cases, $\mathbf{C 1 - C 4}$, as follows: 
C1. If $\left[\mathbf{q}_{21} \cdot \dot{\mathbf{q}}_{1} \geq 0\right] \wedge\left[\mathbf{q}_{21} \cdot \dot{\mathbf{q}}_{2} \geq 0\right]$ : vehicle 2 is before and heading away from vehicle 1 , then

$$
f\left(\mathbf{q}_{1}, \dot{\mathbf{q}}_{1}, \mathbf{q}_{2}, \dot{\mathbf{q}}_{2}\right)=\left\{\begin{array}{c}
1 \text { if } \varphi\left(\mathbf{q}_{21}, \dot{\mathbf{q}}_{1}\right)-\varphi\left(\mathbf{q}_{21}, \dot{\mathbf{q}}_{2}\right) \geq 0 \\
-1 \text { otherwise }
\end{array}\right.
$$

C2. If $\left[\mathbf{q}_{21} \cdot \dot{\mathbf{q}}_{1} \geq 0\right] \wedge\left[\mathbf{q}_{21} \cdot \dot{\mathbf{q}}_{2}<0\right]$ : vehicle 2 is before and heading toward vehicle 1 , then

$$
f\left(\mathbf{q}_{1}, \dot{\mathbf{q}}_{1}, \mathbf{q}_{2}, \dot{\mathbf{q}}_{2}\right)=\left\{\begin{array}{c}
1 \text { if } \varphi\left(\mathbf{q}_{21}, \dot{\mathbf{q}}_{1}\right)-\varphi\left(\dot{\mathbf{q}}_{2}, \mathbf{q}_{12}\right) \geq 0 \\
-1 \text { otherwise }
\end{array}\right.
$$

C3. If $\left[\mathbf{q}_{21} \cdot \dot{\mathbf{q}}_{1}<0\right] \wedge\left[\mathbf{q}_{21} \cdot \dot{\mathbf{q}}_{2}<0\right]$ : vehicle 2 is behind and heading toward vehicle 1 , then

$$
f\left(\mathbf{q}_{1}, \dot{\mathbf{q}}_{1}, \mathbf{q}_{2}, \dot{\mathbf{q}}_{2}\right)=\left\{\begin{array}{c}
1 \text { if } \varphi\left(\mathbf{q}_{12}, \dot{\mathbf{q}}_{1}\right)-\varphi\left(\dot{\mathbf{q}}_{12}, \mathbf{q}_{2}\right)>0 \\
-1 \text { otherwise }
\end{array}\right.
$$

C4. Otherwise (i.e., vehicle 2 is behind and heading away from vehicle 1),

$$
f\left(\mathbf{q}_{1}, \dot{\mathbf{q}}_{1}, \mathbf{q}_{2}, \dot{\mathbf{q}}_{2}\right)=0 .
$$

Notice that vehicle 1 does not turn on the gyroscopic force when vehicle 2 is behind and heading away from vehicle 1 in the detection shell of vehicle 1 .

The energy of each vehicle is given by

$$
E_{i}\left(\mathbf{q}_{i}, \dot{\mathbf{q}}_{i}\right)=\frac{1}{2}\left\|\dot{\mathbf{q}}_{i}\right\|^{2}+V_{i}\left(\mathbf{q}_{i}\right)
$$

with $i=1,2$. One can check that each energy function is non-increasing in time.

Suppose that the initial state $\left(\mathbf{q}_{i}(0), \dot{\mathbf{q}}_{i}(0)\right)$ of each vehicle satisfies

$$
E_{i}\left(\mathbf{q}_{i}(0), \dot{\mathbf{q}}_{i}(0)\right) \leq \frac{1}{2}\left(V_{\max , i}\right)^{2}
$$

with $i=1,2$. We want to show that it cannot happen that the two vehicles collide with $\dot{\mathbf{q}}_{1} \neq 0$ or $\dot{\mathbf{q}}_{2} \neq 0$ at the moment of collision. We prove this by contradiction. Suppose that the two vehicles collided at time $t=t_{c}$ with $\dot{\mathbf{q}}_{1}\left(t_{c}\right) \neq 0$ or $\dot{\mathbf{q}}_{2}\left(t_{c}\right) \neq 0$. Without loss of generality, we may assume that $\dot{\mathbf{q}}_{1}\left(t_{c}\right) \neq 0$. Then, one will reach a contradiction by studying the dynamics of vehicle 1 during the time interval, $\left[t_{c}-\Delta t, t_{c}^{-}\right]$for a small $\Delta t>0$ just as done for the case of obstacle avoidance in $\S 2$. One can also show semi-global convergence of each vehicle to its target point. The proof is almost identical to that in $\S 2$. The point is that each vehicle has its own Lyapunov function which is independent of that of the other vehicle. In this sense, the control scheme given here is a distributed and decentralized control. 


\section{Remarks.}

1. We have not excluded the possibility of zero-velocity collision. Since this happens only at low velocity, one can add an adaptive scheme to handle this.

2. We give another possible choice of the function, $\omega$, in (16). We do this from the viewpoint of vehicle 1 as the same procedure applies to other vehicle. Suppose that vehicle 2 is detected and it does not belong to the case, C4, above. Let $\mathbf{v}_{12}=\dot{\mathbf{q}}_{1}-\dot{\mathbf{q}}_{2}$ be the velocity of vehicle 1 relative to vehicle 2 . We regard vehicle 2 as a fixed obstacle located at $\mathbf{q}_{2}$ and compute $\omega$ with the algorithm used for obstacle avoidance in $\S 2$ with this relative velocity $\mathbf{v}_{12}$.

3 . We give an ad-hoc extension of the gyroscopic collision avoidance scheme to the case of multiple vehicles. The situation is the same as that of two-vehicle case except that there are more than two vehicles. Suppose that vehicle $A$ detected $n$ other vehicles in its detection shell where we have already excluded vehicles which are behind and heading away from vehicle A. Let $d_{i}, i=$ $1, \ldots, n$ be the distance between the safety shell of vehicle A and that of the $i$-th vehicle. Let

$$
\mathbf{q}_{\mathrm{CM}}=\frac{1}{n} \sum_{i=1}^{n} \mathbf{q}_{i}
$$

be the mean position of the $n$ vehicles, and

$$
\dot{\mathbf{q}}_{\mathrm{CM}}=\frac{1}{n} \sum_{i=1}^{n} \dot{\mathbf{q}}_{i}
$$

the mean velocity of the $n$ vehicles. We now design $\omega$ in the gyroscopic force $F_{g, A}=\left(-\omega \dot{y}_{A}, \omega \dot{x}_{A}\right)$ where $\omega$ as follows:

$$
\omega=f \frac{\pi V_{\max }}{\min \left\{d_{i} \mid i=1, \ldots n\right\}}
$$

where one decides the value of $f$ by applying the algorithm used for the two-vehicle case, assuming that there is only one (equivalent) vehicle located at $\mathbf{q}_{\mathrm{CM}}$ with velocity $\dot{\mathbf{q}}_{\mathrm{CM}}$. One needs to modify this in the case that $\mathbf{q}_{\mathrm{CM}}$ coincides with the position of vehicle $A$. The same procedure applies to other vehicles. Simulations show that this ad-hoc scheme works well.

4. If one of the vehicles is "adversarial" then clearly the situation described above needs to be modified.

Simulation: Three Vehicles. Figure 2 shows a simulation of three vehicles. The three vehicles are initially located along the circle of radius 2 and they are $120^{\circ}$ away from one another. The target point of each vehicle is the opposite point on the circle. The detection radius is 0.5 and the safety shell was not considered for simplicity. We used the control law described above with $V_{\max }=$ $\sqrt{2.5}$. The simulation result is given in Figure 2 where the shaded disks denote detection shells. One can see that each vehicle switches on the gyroscopic force when it detects other vehicles. 


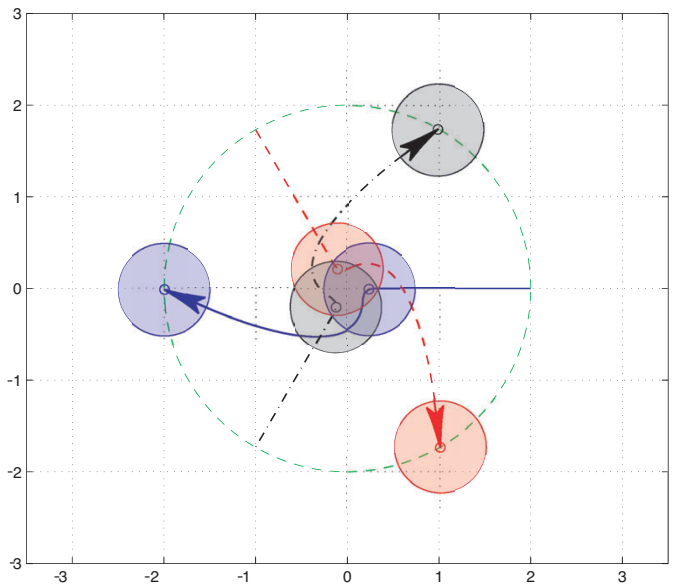

Fig. 2. Three vehicles are initially located along the circle, $120^{\circ}$ away from one another. The target of each vehicle is the opposite point on the circle. The shaded disks are detection shells

\section{Appendix}

One often uses LaSalle's theorem to prove asymptotic stability of an equilibrium in a mechanical system using an energy function $E$. Although LaSalle's theorem is a powerful stability theorem, it normally gives no information on exponential convergence since $\dot{E}$ is only semidefinite. Here, we show that if the energy has a minimum at an equilibrium of interest and the system is forced by a strong dissipative force (and a gyroscopic force), then the equilibrium is exponentially stable. The idea, called Chetaev's trick, is to add a small cross term to the energy function to derive a new Lyapunov function. In this appendix, we give an intrinsic explanation to Chetaev's trick. A preliminary work was done in [2] in a different situation.

Consider a mechanical system with the Lagrangian

$$
L(\mathbf{q}, \dot{\mathbf{q}})=K(\mathbf{q}, \dot{\mathbf{q}})-V(\mathbf{q})=\frac{1}{2} m_{i j}(\mathbf{q}) \dot{q}^{i} \dot{q}^{j}-V(\mathbf{q})
$$

with $\mathbf{q}=\left(q^{1}, \ldots, q^{n}\right) \in \mathbb{R}^{n}$, and the external force, $F=F_{d}+F_{g}$, where $F_{d}$ is a strong dissipation given by

$$
F_{d}(\mathbf{q}, \dot{\mathbf{q}})=-R_{\mathbf{q}} \dot{\mathbf{q}}, \quad R=R^{T}>0,
$$

and $F_{g}$ is a gyroscopic force of the form $F_{g}=S(\mathbf{q}, \dot{\mathbf{q}}) \dot{\mathbf{q}}, \quad S^{T}=-S$. Here we assume that the matrix $S$ is bounded in magnitude on a domain we are interested in. In showing exponential stability, the role of gyroscopic force is little when the dissipation is strong, i.e., $R=R^{T}>0$. Also, one may allow the matrix $R$ to depend on velocity $\dot{\mathbf{q}}$. Here, we use $\mathbb{R}^{n}$ as a configuration space 
for simplicity. However, all arguments will be made in coordinate-independent language in the following.

Suppose the energy

$$
E(\mathbf{q}, \dot{\mathbf{q}})=K(\mathbf{q}, \dot{\mathbf{q}})+V(\mathbf{q})
$$

has a nondegenerate minimum at the origin, $(0,0) \in \mathbb{R}^{n} \times \mathbb{R}^{n}$ : i.e.,

$$
\mathbf{d} V(0)=0, \quad \frac{\partial^{2} V}{\partial q^{i} \partial q^{j}}(0)>0
$$

since the kinetic energy is already positive definite in the velocity $\dot{\mathbf{q}}$. Without loss of generality, we assume that $V(0)=0$.

Let us review the proof of the asymptotic stability of the origin using LaSalle's theorem with $E$ as a Lyapunov function. Consider the invariant subset $\mathcal{M}$ of the set $\{\dot{E}=-\langle\dot{\mathbf{q}}, R \dot{\mathbf{q}}\rangle=0\}$. Suppose $(\mathbf{q}(t), \dot{\mathbf{q}}(t))$ is a trajectory lying in $\mathcal{M}$. Then, $\dot{\mathbf{q}}(t) \equiv 0$. Substitution of this into the equations of motion yields $\mathbf{d} V(\mathbf{q}(t)) \equiv 0$. By (18), the critical point $\mathbf{q}=0$ of $V$ is isolated. It follows $\mathbf{q}(t) \equiv 0$. Hence, $\mathcal{M}$ consists of the origin only. By LaSalle's theorem, the origin is asymptotically stable.

We now devise a trick to show the exponential stability of the origin. Consider the following function $U$ :

$$
\begin{aligned}
U(\mathbf{q}, \dot{\mathbf{q}}) & =E(\mathbf{q}, \dot{\mathbf{q}})+\epsilon \mathbf{d} V_{\mathbf{q}} \dot{\mathbf{q}} \\
& =\frac{1}{2} m_{i j}(\mathbf{q}) \dot{q}^{i} \dot{q}^{j}+V(\mathbf{q})+\epsilon \frac{\partial V}{\partial q^{i}} \dot{q}^{i}
\end{aligned}
$$

with $0<\epsilon \ll 1$. Notice that the definition of $U$ in (19) is coordinateindependent. For a sufficiently small $\epsilon$,

$$
\mathbf{d} U(0,0)=0, \quad D^{2} U(0,0)>0
$$

where $D^{2} U$ is the second derivative of $U$ with respect to $(\mathbf{q}, \dot{\mathbf{q}})$. Hence, $U$ has a minimum at the origin. We will use $U$ as a Lyapunov function. One computes

$$
\frac{d U}{d t}(\mathbf{q}, \dot{\mathbf{q}})=-W(\mathbf{q}, \dot{\mathbf{q}})
$$

where

$$
\begin{aligned}
W(\mathbf{q}, \dot{\mathbf{q}})= & \langle R \dot{q}, \dot{q}\rangle-\epsilon\left(\frac{\partial^{2} V}{\partial q^{i} \partial q^{j}} \dot{q}^{i} \dot{q}^{j}+\frac{\partial V}{\partial q^{i}}\left(-\Gamma_{j k}^{i} \dot{q}^{j} \dot{q}^{k}-\left(m^{-1} \mathbf{d} V\right)_{i}\right)\right) \\
& +\epsilon \frac{\partial V}{\partial q^{i}}\left(\left(m^{-1} R \dot{q}\right)_{i}-\left(m^{-1} S \dot{\mathbf{q}}\right)_{i}\right) \\
= & R(\dot{\mathbf{q}}, \dot{\mathbf{q}})-\epsilon\left(\nabla_{\dot{\mathbf{q}}} \mathbf{d} V\right) \dot{\mathbf{q}}+\epsilon m^{-1}(\mathbf{d} V, \mathbf{d} V)+\epsilon m^{-1}(R \dot{\mathbf{q}}, \mathbf{d} V) \\
& -\epsilon m^{-1}(S \dot{\mathbf{q}}, \mathbf{d} V)
\end{aligned}
$$


where $\nabla$ is the Levi-Civita connection of the metric $m$ and $\Gamma_{j k}^{i}$ are the Christoffel symbols of $\nabla$. One can check that for a sufficiently small $\epsilon>0$

$$
\mathbf{d} W(0,0)=0, \quad D^{2} W(0,0)>0 .
$$

By (20) and (23), there exists $c>0$ such that $\mathbf{d}(W-c U)(0,0)=0$ and $D^{2}(W-c U)(0,0)>0$. Therefore, $W-c U \geq 0$ in a neighborhood $N$ of the origin. This and (21) imply $\frac{d U}{d t} \leq-c U \leq 0$. It follows

$$
U(\mathbf{q}(t), \dot{\mathbf{q}}(t)) \leq U(\mathbf{q}(0), \dot{\mathbf{q}}(0)) e^{-c t}
$$

on $N$. This proves the exponential stability of the origin. One can also go further by invoking the Morse lemma to find a local chart $z=\left(z^{1}, \ldots, z^{2 n}\right)$ in which the function $U$ becomes $U(z)=\sum_{i=1}^{2 n}\left(z^{i}\right)^{2}$. Hence, (24) implies $\|z(t)\| \leq\|z(0)\| e^{-\frac{c}{2} t}$ where $\|z\|=\sqrt{\sum_{i=1}^{2 n}\left(z^{i}\right)^{2}}$.

Acknowledgements. We thank Noah Cowan, Sanjay Lall, Naomi Leonard, Elon Rimon, Clancy Rowley, Shawn Shadden, Claire Tomlin, and Steve Waydo for their interest and valuable comments. Research partially supported by ONR/AOSN-II contract N00014-02-1-0826 through Princeton University.

\section{References}

1. Bhatta, P., Leonard, N. (2002) "Stabilization and coordination of underwater gliders", Proc. 41st IEEE Conference on Decision and Control.

2. Bloch A., Krishnaprasad P.S., Marsden J.E., Ratiu T. (1994) Annals Inst. H. Poincaré, Anal. Nonlin., 11:37-90.

3. Chang D.E., Bloch A.M., Leonard N.E., Marsden J.E., Woolsey C. (2002) ESAIM: Control, Optimization and Calculus of Variations, 8:393-422.

4. Chang, D.E., Shadden S., Marsden, J.E. and Olfati-Saber, R. (2003), Collision avoidance for multiple agent systems, Proc. CDC (submitted).

5. Hwang I., Tomlin C.(2002) "Multiple aircraft conflict resolution under finite information horizon", Proceedings of the AACC American Control Conference, Anchorage, May 2002, and "Protocol-based conflict resolution for air traffic control", Stanford University Report SUDAAR-762, July, 2002.

6. Jadbabaie A, Lin J, Morse A.S. (2002) "Coordination of groups of mobile autonomous agents using nearest neighbor rules", Proc. 41st IEEE Conference on Decision and Control, 2953-2958; see also IEEE Trans. Automat. Control (to appear 2003).

7. Kosecká, J., Tomlin C., Pappas, G., Sastry, S. (1997) "Generation of conflict resolution maneuvers for air traffic management", IROS; see also IEEE Trans. Automat. Control, 1998, 43:509-521.

8. Rimon E., Koditschek D.E. (1992) IEEE Trans. on Robotics and Automation, $8(5): 501-518$.

9. Wang L.S., Krishnaprasad P.S. (1992) J. Nonlinear Sci., 2:367-415. 